\title{
Gubernamentalidad y agua: Analíticas del poder en el desierto de Atacama ${ }^{1}$
}

\section{Governmentality and water: Analytics of power in the Atacama desert}

Recibido: 27 de agosto de 2015 - Revisado: 29 de marzo de 2016 - Aceptado: 07 de mayo de 2016

\section{Alfredo García Carmona ${ }^{2}$}

\section{Resumen}

¿Puede contribuir la filosofía política propuesta por Michel Foucault a la comprensión, explicación y transformación de la crisis hídrica actual en el norte de Chile? Revisando el concepto foucaultiano de gubernamentalidad, se propone contribuir al enriquecimiento de un marco teórico crítico con el fin de advertir cómo, en el contexto del capitalismo neoliberal, grandes corporaciones y mega-proyectos están interviniendo en la destrucción de ecosistemas específicos y microterritoriales de Chile, tomando como caso específico el desierto de Atacama y la cuestión del agua.

El interés principal es examinar cómo el concepto de gubernamentalidad ayuda a comprender las modalidades que asume el ejercicio de poder, siendo una de ellas los gobiernos políticos que normalizan situaciones de deterioro de la naturaleza. Se expondrá, específicamente, el caso del agua en la región de Atacama, Chile.

\section{Palabras clave}

Gubernamentalidad, agua, neoliberalismo, Atacama, Chile.

\begin{abstract}
Can the political philosophy proposed by Michel Foucault contribute to the understanding, explanation and transformation of the current water crisis in northern Chile? Reviewing the Foucaultian concept of governmentality, the article intends to contribute to the enrichment of a critical theoretical framework in order to notice how, in the context of neoliberal capitalism, large corporations and mega-projects are intervening in the destruction of specific and microterritorial ecosystems of Chile, taking as a specific case the Atacama desert and the water issue.

The main interest is to examine how the concept of governmentality helps to understand the modalities assumed by the exercise of power, one of them being political governments that normalize situations of deterioration of nature. The case of water in the Atacama region, Chile will be specifically discussed.
\end{abstract}

\section{Keywords}

Governmentality, water, neoliberalism, Atacama, Chile.

\footnotetext{
${ }^{1}$ Ensayo de reflexión teórica de la Universidad de Atacama, Chile.

2 Sociólogo de la Universidad de Concepción, Magister en Antropología y Desarrollo de la Universidad de Chile. Profesor Instructor e investigador de la Facultad de Ciencias Jurídicas y Sociales de la Universidad de Atacama en Chile. Becario Comisión Nacional de Ciencia y Tecnología- CONICYT Chile.

Correo electrónico: alfredo.garcia@ uda.cl

Para citar este artículo use: García, A. (2017). Gubernamentalidad y agua: Analíticas del poder en el desierto de Atacama. Civilizar Ciencias Sociales y Humanas, 17(33), 113-134. doi: $10.22518 / 16578953.903$
} 


\section{Introducción}

El capitalismo mundial integrado (Guattari, 2015) nos sitúa en un contexto geopolítico completamente distinto al de décadas pasadas; esta fase del capitalismo postindustrial ha sido frecuentemente llamada neoliberal, y se caracteriza por otorgar al mercado un rol regulador de la economía y porque las sociedades están cada vez más interconectadas en el planeta.

En este marco, el neoliberalismo es:

Ante todo, una teoría de prácticas políticoeconómicas que afirma que la mejor manera de promover el bienestar del ser humano consiste en no restringir el libre desarrollo de las capacidades y de las libertades empresariales del individuo, dentro de un marco institucional caracterizado por derechos de propiedad privada, fuertes mercados libres y libertad de comercio. El papel del Estado es crear y preservar el marco institucional apropiado para el desarrollo de estas prácticas (Harvey, 2007, p. 8).

Esta definición, de enfoque principalmente marxista, es fundamental para entender (en términos económicos) cómo en Latinoamérica y concretamente en Chile, la apertura de la economía, mediante una serie de políticas económicas instauradas por las dictaduras de la región establecidas a partir de las décadas de los 70 y 80 , permitieron que las inversiones de transnacionales extranjeras pudieran instalar sus proyectos en distintos rubros, principalmente extractivos.

Maristella Svampa (2013), indica que este modelo económico productivo progresará en posteriores gobiernos, postdictatoriales algunos de ellos, y será el resultado del "consenso de los commodities", en donde se establece como forma de gobierno a nivel global, liderada principalmente por Estados Unidos, lo que permitirá el establecimiento de los marcos jurídicos e institucionales internacionales para la extracción de los bienes comunes naturales de nuestra región.
Esto es explicado por Harvey (2005) en su libro El nuevo imperialismo (The new imperialism); en el que señala que existe una fórmula medular denominada "acumulación por desposesión”, donde las economías centrales reajustarían espacio-temporalmente los excedentes de la sobreacumulación de sus economías. Por ejemplo, en nuestro continente, mediante la inversión en megaminería y agroindustria. Para el autor, es una forma de acumulación originaria, que para nuestro caso explica la explotación extractiva de minerales y el apoyo del complejo "Wall Street-Reserva Federal-FMI dentro de Estados Unidos, capaz de controlar las instituciones globales y de proyectar un vasto poder financiero alrededor del mundo mediante una red de otras instituciones financieras y gubernamentales, [que] ha jugado un rol determinante y problemático en la dinámica del capitalismo global en los años recientes" (Harvey, 2005, p. 110).

Así, con el paso de los años, las industrias extractivas de minería y agroindustria han causado una serie de efectos tanto en el entorno natural como en los sistemas de vida de comunidades locales. El caso de la minería es emblemático pues efectivamente refleja cómo las empresas transnacionales, desde hace siglos, han ocasionado una serie de problemas socioambientales en nuestra región (Machado, 2014), los cuales se han incrementado notablemente en las últimas décadas con el surgimiento de esta nueva fase del capitalismo (CEPAL, 2015; WWAP, 2006).

La economía chilena se caracteriza por una fuerte operación minera, la cual es muy importante para el PIB nacional; sin embargo esa actividad ha ido ocasionando, específicamente en el norte del país, una serie de efectos problemáticos, como contaminación de aguas y escasez de recursos hídricos.

Si bien existen argumentos económicos como el nivel de producción, ligados al aumento de inversión extranjera, las disminución de 
costos de operación, las externalidades negativas, entre otros, para explicar los efectos sobre el medio ambiente fruto de actividades productivas, es interesante observar qué rol juegan las instituciones gubernamentales locales, pues a través de los marcos jurídicos e institucionales estatales es que se normalizan estas conductas empresariales en función del crecimiento económico y el desarrollo, que son elementos claves del discurso del modelo neoliberal.

Esta estabilización en la normalidad, en definitiva, ha hecho que

El neoliberalismo se ha[ya] tornado hegemónico como una forma de discurso, [el cual] posee penetrantes efectos en los modos de pensamiento, hasta el punto de que ha llegado a incorporarse a la forma natural en que muchos de nosotros interpretamos, vivimos y entendemos el mundo (Harvey, 2007, p. 9).

Pero como la producción de subjetividad en contextos geográficos específicos de estos discursos, así como su forma de operar en las temáticas ambientales no se ha estudiado en profundidad, el presente artículo permitirá entender cómo es que en el desierto más árido del mundo se naturalice y acepte un uso irracional del agua, lo que está provocando su escasez.

Un interesante análisis de la forma en que estos discursos se han establecido y se ven reflejados en la manera de actuar de Estados, empresas y sociedad civil a nivel global, es la arqueología del tratamiento de la cuestión ambiental en los documentos del Sistema de Naciones Unidas, realizada por José Seoane (2015); este estudio, que extrae los principales enunciados de distintos documentos de esta red de organizaciones, establece cómo configuran, a lo largo de décadas, lo que Leff (2006) denominó el "paradigma de la capitalización de la naturaleza".

Tal paradigma supone "la expansión y profundización de un proceso de mercantilización de los bienes naturales, el ambiente, la na- turaleza y la vida en todas sus formas" (Seoane, 1995, p. 152). Para el autor,

La emergencia del desarrollo, de estas reflexiones sobre la práctica de gobierno de la cuestión ambiental, se inscribió en una serie de transformaciones en el terreno de las políticas públicas a nivel local y nacional, y de los acuerdos, las instituciones y las regulaciones a nivel global. Ese proceso se cristalizó, entre la década de los 80 y la primera década del actual siglo, en la constitución de ciertos campos temáticos conformados alrededor de términos como 'contabilidad ambiental', 'capital natural', 'servicios ambientales', 'mercado de carbono', 'economía verde' y 'gobernanza ambiental', donde se articularon una serie de prácticas discursivas y extradiscursivas en el tratamiento neoliberal de la cuestión ambiental (p. 147).

Ahora bien, en cuanto al tratamiento del tema ambiental, se han desarrollado diversas segmentaciones que operan sobre el agua, el aire, el suelo, el mar, los glaciares, entre otros. En lo que respecta al agua, el asunto ha cobrado mucho interés cuando distintos organismos internacionales como UNESCO y CEPAL, han desarrollado programas que han profundizado en el diagnóstico de las problemáticas del agua en el mundo y en Latinoamérica, respectivamente. Esto se aprecia a través de distintos estudios, como el de Water Resources Group, que asegura que en "2030 el mundo tendrá que enfrentarse a un déficit mundial del 40\% de agua" (2030 WRG, 2009 en WWAP, 2015). Sin embargo, es posible señalar que, si bien hay una mayor demanda del consumo del agua, principalmente por parte de la industria así como de la población en aumento, "la crisis hídrica mundial es una crisis de gobernanza" (WWAP, 2006), "más que una crisis de escasez" (WWAP, 2015).

En este orden de ideas, el objetivo de este artículo es evidenciar que "el gobierno del agua" es posible analizarlo mediante el concepto de gubernamentalidad, el cual opera como "conducción de conductas" respecto al agua. 
Además, y como complemento de una explicación netamente económica, es posible agregar que en el contexto neoliberal los mecanismos de subjetivación y producción de subjetividad son un pilar fundamental.

\section{El gobierno neoliberal del agua en Chile}

Chile es un caso emblemático de aplicación del modelo neoliberal en el mundo (Murillo, 2015; Palominos, 2015): a partir del denominado "experimento Chile" se instaura un modelo societal, que venía fraguándose a escala global desde el siglo XIX (Murillo, 2015), y cuyos principales elementos son: la libertad de mercado, la privatización de la economía, la prioridad del derecho a la propiedad privada y la disminución del tamaño del Estado.

Después de la Segunda Guerra Mundial y con el auge de Estados Unidos como potencia imperial (Quijano, 2002), asistimos a una nueva fase del capitalismo donde Chile, a través del golpe de Estado y la posterior dictadura cívico-militar (1973-1989), implementa una serie de políticas económicas basadas en los discursos de la doctrina económica y social de la "escuela de Chicago", a cargo de su mentor Milton Friedman, considerado el padre del neoliberalismo estadounidense (CEP, 1992).

Con la dictadura cívico-militar (19731989) se instala un modelo económico neoliberal que genera una privatización de los recursos naturales (bosques, agua, minerales y recursos marinos) promoviendo la apertura de los territorios para la inversión de capitales internacionales interesados en la agricultura extensiva, forestal y la megaminería. De tal modo, se comienza a presentar un crecimiento económico que trae consigo una serie de problemas en el entorno local, relacionados con alteraciones en los sistemas de vida y el deterioro de la naturaleza. Así, junto con el aumento en los indicadores macroeconómicos del país, los problemas de escasez de recursos hídricos, polución del aire debido a material particulado, contamina- ción por desechos industriales, entre otros, van generando un malestar en la población y los ciudadanos comienzan a exigir, poco a poco soluciones a estas problemáticas. Con el regreso en Chile de la democracia en 1990, las personas son conscientes de que es posible que tengan una mayor injerencia en estos temas.

Sin embargo, el asentamiento del modelo económico neoliberal, durante las siguientes décadas, se profundizará por las normativas jurídicas establecidas en la Constitución Política de 1980, el Código de Aguas de 1981 y el Código de Minería de 1983, entre otras, que actualmente continúan siendo los principales marcos jurídicos que norman estas actividades.

En 1981, la dictadura cívico-militar instala en Chile un modelo de gestión privada del agua que plantea un paradigma neoliberal radical (Prieto, 2015). Dicho patrón se sustenta tanto en la Constitución de 1980 como en el Código de Aguas de 1981. A partir de estos documentos matrices se constituye la institucionalidad estatal que administrará el agua en el país.

Lo que vino a instaurar este modelo fue la entrega del agua, su administración y gestión a los privados, relegando al Estado, que otrora fuese el actor principal. Esto, muy en línea con la política económica del neoliberalismo (CEP, 1985): neutralizando el poder estatal, estableciendo el derecho a la propiedad como fundamento, instaurando la regulación de la oferta y la demanda, y creyendo que el libre mercado asegura que los recursos fluyan eficientemente. En síntesis, el "agua [es] reducida a una mercancía” (Prieto, 2015, p. 150), y es objeto de derechos de aprovechamiento, lo que la hace susceptible de apropiación privada $\mathrm{y}$ de libertad de transacción en el mercado (Prieto, 2015).

A partir de la gestión de la Dirección General de Aguas (DGA), se comienzan a otorgar los derechos de aprovechamiento con los mínimos exigidos por la ley, y demostrándose 
una serie de problemas, donde la capacidad normativa de la institución para planificar usos y resolver conflictos, es deficitaria (Prieto, 2015). El Código de Aguas, en sus Artículos 13 y 14, establece que hay derechos de agua consuntivos y no consuntivos; los primeros son los que se usan a cabalidad en cualquier actividad, y los segundos son los que se usan y luego se devuelven (por ejemplo, en el caso de las hidroeléctricas). Toda esta forma de administración de mercado va a generar una acumulación de agua por parte de quienes detentan mayor poder económico, al estar sujetas a las leyes de oferta y demanda (Duarte \& García, 2013; Torres \& García, 2009). En el desierto de Atacama, debido a una mayor demanda y a una menor oferta del agua como mercancía, se va a producir una competencia entre privados, la cual, de acuerdo con el poder adquisitivo de unos y otros, favorecerá a la minera y a la agroindustria, en detrimento de otras actividades.

De este modo,

El proceso de regularización de los derechos de propiedad sobre los derechos de agua escapa a toda pretensión de espontaneidad pretendida por los discursos neoliberales, [aquí] la DGA implementó un sistema de regularización basado en la simplificación, con el propósito de generar visibilidad y, así, el control del recurso hídrico (Prieto, 2015, p. 155).

En consecuencia, va a ser "el sistema político-institucional el que, enraizado en discursos utilitaristas y pretensiones de economía política, favorece la propiedad del agua y su flujo de acumulación en ciertos rubros" (Prieto, 2015, p. 161; Torres \& García, 2009).

\section{La "caja de herramientas" de Foucault}

Para este análisis, se realizará un acercamiento teórico a los conceptos suministrados por la "filosofía analítica del poder", postulada por Michel Foucault (Castro, 2004; Foucault, 2015), la cual se aplicará específicamente en te- máticas ambientales. Esto, según la definición de su trabajo como una "caja de herramientas" (Morey, 2014), cuya finalidad es ser apropiada, utilizada y criticada en función de un ejercicio intelectual que sea proyectado para estudiar los ejercicios de poder, saber y subjetivación.

La analítica del poder consiste en explicar los fenómenos en función del ejercicio de poder sobre los cuerpos de un determinado espacio o lugar. Con esto, Foucault entiende el poder como una relación, como parte constitutiva de toda relación social, y lo definirá en términos generales como la forma de "conducir conductas y disponer de su probabilidad induciéndolas, apartándolas, facilitándolas, dificultándolas, limitándolas, impidiéndolas" (Castro, 2004, p. 412). Esta amplia definición y el enfoque sobre las dinámicas del ejercicio del poder, lo alejarán de una visión más estructural, que entiende el poder principalmente como ejercicio de dominación y explotación; para Foucault, no es negar dicha perspectiva, sino complementarla o ampliarla en términos de cómo este ejercicio de poder es principalmente histórico y deviene en sus estrategias mutando constantemente, por lo cual debiéramos ceñirnos a su análisis exhaustivo en las prácticas discursivas y extra discursivas en contextos específicos.

Por otra parte, el método de Foucault, denominado arqueológico y genealógico, va a consistir, por tanto, en analizar las formas del ejercicio del poder a lo largo de la historia occidental. Aun así, él mismo aclarará que le interesa focalizarse en cómo este poder construye un sujeto, su finalidad es una historia de la subjetividad (Morey, 2014), en donde la arqueología se enfocará en el saber, que contribuye a esta "forma-sujeto"; y la genealogía se concentrará en los ejercicios de poder que construyen dicha subjetividad necesariamente histórica.

Aplicar estos conceptos a temas ambientales no es nuevo. Se han desarrollado muchos trabajos que han aprovechado las categorías propuestas por Foucault al análisis de temáti- 
cas ambientales, de los cuales citaremos los siguientes: Agrawal (2006) propone la definición de sujetos ambientalistas para entender la construcción de subjetividad que conforma un individuo dentro de la red de poder de gubernamentalidad ambiental; Rutherford (2007), por su parte, adelantó un análisis de diversas categorías como el poder, el saber, el ethos y la subjetividad, en su aplicación a temas ambientales; Bakker (2010) definió un análisis de las instituciones y ejercicios de poder global en el ambientalismo de mercado del agua; y Swyngedouw (2015), desarrolló el concepto de "ciclo hidrosocial" como un ejercicio de poder híbrido entre los sistemas sociales y sistemas hídricos.

Stephanie Rutherford (2007), profesora de Estudios del Medioambiente, en su artículo titulado "Green governmentality: insights and opportunities in the study of nature's rule" (Gubernamentalidad verde), expone una aplicación específica de la gubernamentalidad a los temas ambientales, y considera fundamentales, para el entendimiento de la gubernamentalidad como forma de gobierno en torno a los temas ambientales, el poder, el biopoder y la construcción de subjetividad. Respecto al poder, analiza los flujos presentes en todas las relaciones sociales, más allá de la dicotomía gobernador/ gobernado, entendiendo que hay instituciones, prácticas, hábitos, que también pueden ser estudiados para entender el funcionamiento del poder (espacios micropolíticos). Siguiendo a Foucault, plantea que el poder no es formal, sino que es sustancial, se ejerce y se resiste dependiendo del contexto histórico. También le interesa lo que Foucault denominó como biopoder, que es una forma de poder sobre la vida y las poblaciones de manera global, es decir, la posibilidad de entender el objetivo del poder volcado sobre la población, más que sobre el individuo. Y finalmente, la autora considera que la construcción de subjetividad es la menos estudiada de las dimensiones del concepto de gubernamentalidad, pero quizás la más importante, pues a partir de ella se entenderá que el poder opera desde dentro de los sujetos, quienes se gobiernan a sí mismos mediante mecanismos de subjetivación gatillados por distintos dispositivos de poder.

Así, el concepto de gubernamentalidad como potente herramienta de análisis, ha desembocado entonces, en diversas versiones: gubernamentalidad verde, environmentality (Luke, 1995); gubernamentalidad aplicada a lo ambiental (Darier, 1999); y en América Latina, la ecogubernamentalidad planteada por Ulloa (2011), quien la ha aplicado concretamente para entender la forma sujeto de las poblaciones indígenas en Colombia y su inserción en la economía global, a partir de diversos ejercicios de poder gubernamental global en temas ambientales.

En el mismo camino y también en Colombia, Santiago Castro Gómez (2010) en su obra "Historia de la Gubernamentalidad", ha hecho una revisión exhaustiva del concepto, objeto del presente artículo. Castro-Gómez explica el contexto del nacimiento de la reflexión por parte de Michel Foucault en sus cursos del Collège de France (1978-1979), analiza sus principales conceptos teóricos, así como los pasos que siguieron sus adeptos al deceso del filósofo. Sin embargo, el presente trabajo intenta hacer una aplicación microterritorial de esta serie de herramientas analíticas, más que una revisión teórica de la obra de Foucault y de la que de ella se ha derivado.

Para el análisis se utilizará el concepto de gubernamentalidad, que Foucault definió como

El conjunto constituido por las instituciones, los procedimientos, análisis y reflexiones, los cálculos y las tácticas que permiten ejercer esta forma bien específica, aunque muy compleja, de poder que tiene por blanco principal la población, por forma mayor de saber la economía política y por instrumento técnico especial los dispositivos de seguridad" (Foucault, 2006, p. 136).

Si bien Foucault (2006) también atribuyó este fenómeno a "una línea de fuerza" en occi- 
dente en general y producto de una etapa anterior de "estado de justicia de [la] Edad Media" (p. 136), escapa de este análisis ese devenir de más larga data de este concepto hasta nuestros tiempos y su reterritorialización en América Latina.

Ahora bien, el presente estudio propone complementar dicha definición de gubernamentalidad con una precisión y un énfasis en el fenómeno de la subjetivación y las resistencias, propias de esta forma específica y compleja de poder. Siguiendo a Foucault (2007) se entiende la subjetivación como esa forma de constitución del sujeto en donde este genera "actividades sobre sí mismo", y en donde las resistencias pueden ser entendidas como luchas, en particular el tercer tipo de lucha que considera Foucault (1994), que es la "lucha de sujeción", es decir la resistencia a estas formas de objetivación del sujeto, definidas por particulares formas de gobierno.

\section{Gubernamentalidad y agua en el desierto de Atacama}

Para entender y plasmar la complejidad de los fenómenos de las problemáticas ambientales en general, y al agua en particular, se requieren renovar las herramientas conceptuales. Por lo tanto, entender la gubernamentalidad como herramienta analítica, permite analizar la forma de gobierno específica, con respecto a la cuestión del agua, que se da en el desierto de Atacama, y cuáles son las particularidades que presenta el gobierno del agua en el desierto más árido del mundo, bajo un modelo neoliberal como el chileno.

La región de Atacama, una de las quince en las cuales se divide el Estado nacional unitario de Chile, se encuentra en el norte del país $\left(\sim 26^{\circ} \mathrm{S}-29^{\circ} \mathrm{S}\right)$ y cuenta con una población de 290.581 habitantes (INE, 2012). Su estructura político-administrativa es una representación regional del poder central del Estado, es decir, por cada Ministerio nacional (de Minería, Me- dio Ambiente o Economía, entre otros), existe una Secretaría Regional Ministerial (SEREMI) que representa el poder del ministro nacional en las regiones. Al interior de cada Ministerio se alojan diversos servicios específicos, como la Dirección Nacional de Aguas, que también poseen oficinas regionales.

Al estar en pleno desierto de Atacama, las tres cuencas hidrográficas albergan las zonas residenciales, industriales y comerciales de la región, que configuran el hábitat humano de los atacameños. Aquí coexisten diversas actividades productivas, pero sin duda la minería y la agricultura son las de mayor envergadura.

El desierto de Atacama es un ecosistema caracterizado por la poca cantidad de agua que requiere para su equilibrio natural, con una precipitación que no supera los $18 \mathrm{~mm}$ al año para toda la región (Squeo, Arancio, \& Gutiérrez, 2008, p. 26). Desde la década de 1980 y a raíz de las actividades productivas, se observan cambios considerables en el entorno natural, es decir, una serie de problemas socioambientales; para efectos de este artículo y por ahora, estos problemas serán interpretados como las afecciones en la población producto de alteraciones en sus entornos naturales, y que han sido provocados por el ser humano.

Aun cuando las tres grandes cuencas hídricas de la región son distintas, es posible evidenciar que al interior de estas existe una tensión respecto al uso del agua y pugnas entre los diversos actores, lo cual se transforma en una acumulación de agua para fines productivos de los privados con mayor poder adquisitivo (grandes empresarios). En la cuenca del Chañaral, la contaminación del río Salado es uno de los mayores hitos históricos de contaminación de aguas en el país; esto se debió a los desechos de la minería de cobre que, entre 1939 y 1988, la Corporación Nacional del Cobre (Codelco), empresa minera estatal, vertió sobre el cauce (Cortés, 2010). Por su parte, en la cuenca del río Copiapó es posible señalar 
como dato relevante, que, durante el proceso de otorgamiento de derechos de aprovechamiento, la Dirección General de Aguas en la región entregó muchas más acciones de agua de las que tenía el río para su reabastecimiento; esto se debió a la presión ejercida para ejecutar los proyectos productivos, mineros y agroindustriales, en la región. (Delegación Presidencial para los Recursos Hídricos, 2015). Y en la cuenca del Huasco, la irrupción del proyecto minero Pascua-Lama, ha dado inicio al conflicto por el uso del agua entre agricultores, mineros y comunidades campesinas (Torres \& García, 2009, Urkidi, 2015). A ello se suma que, en los últimos años, han ido aumentando los problemas de suministro y calidad del agua potable, por lo que la empresa privada responsable de dicho proyecto ha debido enfrentar una serie de sanciones por parte de la Superintendencia de Servicios Sanitarios (SISS).

En general, en las tres cuencas existe una tensión, un juego de poder distintivo entre los diversos actores, lo cual favorece a algunos en detrimento de otros. En este sentido, el aporte que hace el concepto de gubernamentalidad al análisis de las relaciones de poder, es entenderlas como una red de fuerzas policéntricas que atraviesan los enlaces sociales a través de todo el tejido social, y ya no más solo desde una dicotomía gobernador/gobernado.

Para hablar de la gubernamentalidad y el agua en el desierto de Atacama, se ha de entender que allí se configura una forma de gobierno muy particular (en cuanto al agua se refiere), debido al contexto territorial de su expresión. Para comprender los complejos ejercicios de poder que atraviesan a quienes cuyas conductas responden al uso del agua, es necesario acercarse y detallar cada uno de los distintos elementos que, heterogéneamente, se relacionan para ejercer este poder sobre una población determinada, sustentándose en la economía política como saber fundamental y con dispositivos de seguridad especializados (Foucault, 2007).
Para Foucault, los elementos constitutivos de la gubernamentalidad son principalmente seis: instituciones, procedimientos, análisis, reflexiones, cálculos y tácticas.

a) Instituciones. Se pueden clasificar en función de su nivel de injerencia en el ejercicio de conducir la conducta de las personas en torno al uso del agua. En primer lugar, se encuentra la Dirección General de Aguas (DGA); después, las encargadas de la gestión $\mathrm{y}$ administración del agua en un territorio o cuenca específico: las Juntas de Vigilancia de agua y las Asociaciones de Agua Potable Rural (APR). Estas instituciones son las encargadas de disponer los procedimientos de uso del agua que establece el Código de Aguas de 1981.

En otro nivel, existen instituciones político-administrativas como las Intendencias Regionales, Gobernaciones Provinciales y las Municipalidades comunales, que son las instituciones territoriales cuya función no está directa ni exclusivamente relacionada con el uso del agua, pero dependiendo del caso, intervienen principalmente en la resolución de conflictos.

Otros actores importantísimos en esta red de instituciones son las empresas privadas; en Chile, paulatinamente, las empresas sanitarias, antes estatales, han devenido privadas por tener mayoritariamente capitales privados y transnacionales. En relación con el caso de estudio, la empresa sanitaria de la región de Atacama ha cobrado notoriedad pública debido a reiterados casos de problemas en la entrega del servicio por calidad del agua y cortes no programados.

Se encuentra también la Superintendencia de Servicios Sanitarios, que es el organismo nacional, con oficinas regionales, que se encarga de fijar las tarifas, supervisar las empresas, otorgar concesiones, entre otras funciones, y es quien ha sancionado a la empresa sanitaria de la región. 
Por otra parte, está la Dirección de Obras Hidráulicas, perteneciente al Ministerio de Obras Públicas, que se encarga de todas las obras gruesas de infraestructura hidráulica: embalses, contenciones, pasos, alcantarillados, entre otros.

Por último, están los organismos regionales, representantes del Ministerio del Medio Ambiente, que en este territorio tienen sus dependencias locales alojadas en la Secretaría Regional Ministerial de Medio Ambiente. Estos organismos operan a través del Servicio de Evaluación Ambiental (SEA), el Servicio de Biodiversidad y Áreas Protegidas, y los Tribunales Ambientales.

Al respecto, añádase que el poder judicial en general, y los Tribunales Ambientales en particular (de reciente creación en el año 2012), son relevantes debido a los procesos judiciales que se han instaurado para solucionar varios conflictos sociales derivados del uso del agua en el país.

b) Procedimientos. Se puede observar que están establecidos en una serie de documentos que son los que orientan la acción de los sujetos en su calidad de ciudadanos y consumidores. Un grupo de documentos son los que, desde el marco jurídico y administrativo, establecen cómo administrar y gestionar el agua (recurso). Partiendo de un nivel macro, son: la Constitución Política de la República, Chile de 1980; el Código de Aguas, de 1981; el Código de Minería, de 1983; la Ley de Bases del Medioambiente, de 1994; la Ley del Ministerio de Medioambiente (2010); y la Ley Indígena, entre otros.

Por otra parte, para los procedimientos que establecerán, en diferente nivel, las formas de conducirse respecto al tema del uso del agua, se haya el conjunto de documentos de corte formalmente político, pues son los ordenamientos que establecen los gobiernos de turno, y que se asientan sobre las bases jurídicas mencionadas anteriormente: políticas públicas, programas, proyectos, estrategias de gobiernos formales de turno, entre otros.

De igual modo, se observan los procedimientos que se establecen al interior de cada empresa de acuerdo con sus políticas y protocolos respecto al uso del agua. Así mismo, están los procedimientos establecidos al interior de las organizaciones de gestión del agua como las Juntas de vigilancia de agua, las asociaciones de agua potable rural, las comunidades de agua, entre otras.

Terminando, pero no por ello menos importantes, están las organizaciones de consumidores, recientemente constituidas, y que en la región establecen también procedimientos que tienen que ver con la supervisión de las formas del servicio de agua, el cual, por muchos años ha presentado deficiencias.

Así mismo, los organismos y servicios públicos también suponen otra serie de procedimientos en cuestión.

c) Análisis. Tal como lo plantea Legg (2005), hay formas de conocimiento que posicionan un nuevo "régimen de verdad" (Foucault, 2007) por sobre otro régimen vigente. Respecto al régimen de verdad con la cuestión del agua en el desierto, se pueden establecer dos grandes ejes. En primer lugar, la forma de establecer, desde la construcción del conocimiento, la relación sociedad/naturaleza, construcción de conocimiento que contribuye a cierto de régimen de verdad, se fundamenta en la separación entre el sujeto y el objeto, propio del paradigma positivista de inicios de la modernidad (Lander, 2010), con lo cual se deja fuera otro tipo de epistemes, que no realizan esta escisión hombre/naturaleza, como las de ciertas comunidades indígenas.

En segundo lugar, las formas de establecer el gobierno sobre los bienes comunes naturales, enunciados esencialmente en los prin- 
cipales documentos discursivos jurídicos y legales, tales como la Constitución Política de la República, el Código de Aguas y el Código de Minería. Es a través de estas normativas que el régimen dictatorial (1973-1989) utilizó como saber fundamental la economía política para establecer las bases sobre las cuales se entenderán los bienes comunes como "recursos naturales", por ende, transables del mercado. De lo anterior se derivan una serie de análisis econométricos que se pueden subdividir en políticos, financieros, físico-naturales y sociales.

En otro orden de ideas, los estudios que dan cuenta de la representación de la realidad que se establece en torno al ambiente y al agua, poseen varias peculiaridades. Por una parte, los estudios desarrollados (caudal, glaciares, etc.) son mucho más recientes que la instalación de proyectos, lo que indica que se han ido realizando en función de la toma de conciencia de la falta de estos para alcanzar decisiones acertadas en términos ambientales. Por otra parte, la mayoría de los estudios son adelantados por empresas o centros especializados privados, y en menor medida, el Estado se está haciendo cargo de producir información y conocimiento en estas relevantes áreas.

Por otra parte, en distintos organismos internacionales han proliferado las investigaciones que atañen a las formas de establecer un "buen gobierno" de los recursos ambientales y del agua. Un ejemplo de ellos son los estudios adelantados por la ONU y CEPAL, que además de acuñar los conceptos de gobernabilidad y gobernanza ambiental, proponen que se mantenga la estabilidad de los modelos económico-productivos de la región, y aportan directrices respecto a las tecnologías políticas de gobierno para resolver eventuales conflictos socioecológicos, estableciendo medidas dentro de los marcos jurídicos ya existentes.

Además, existe una representación de la realidad que se construye sobre la pugna entre el conocimiento experto frente a los saberes populares. Se entiende que hay un conocimiento hegemónico que instala una imagen de realidad externa que se contradice con otro tipo de conocimiento no hegemónico. Esto es observable en los movimientos de resistencia que cuestionan estas imágenes construidas por las instituciones hegemónicas y que plantean una controversia debido a distintos lenguajes de valorización (Martínez, 2004).

d) Reflexiones. Estos distintos lenguajes se pueden observar en los razonamientos surgidos en el presente trabajo. Cuando Foucault clasifica a las reflexiones como uno de los elementos de la gubernamentalidad, es posible inferir que hay enunciados que no exigen la formalización de un análisis, como si lo requerirían los de un estudio científico especializado, pero que por su circulación es posible observar que permean los discursos de los actores involucrados en estas cuestiones. $\mathrm{La}$ importancia de las reflexiones de distinto tipo radica en la construcción de realidad, imagen aprendida que se constituye en los discursos de los sujetos.

Dado lo anterior, vale la pena indicar que existen reflexiones que no se encuentran en documentos oficiales o institucionalizados, pero que se expresan en discursos, reuniones, conversaciones informales, entre otras. Las reflexiones más significativas para el caso de estudio, y que surgen en los espacios de cotidiana discusión de temas ambientales y sociales en la región de Atacama, son "el agua es vida" y "el agua vale más que el oro"; estas dos frases, que se escuchan en las marchas de protesta contra el proyecto Pascua-Lama, sintetizan las ideas y opiniones dispersas de un grupo de personas que cuestionan las formas en que se construye el "valor" del agua, más allá que una mercancía o capital económico.

e) Cálculos. En cuanto a ellos se refiere, se observa cómo los distintos actores los aplican para estimar diferentes criterios. Las 
empresas calculan sus acciones en función de las ganancias, inversiones, impactos y costos de operación, estos serán los parámetros que guiarán sus acciones. Por otra parte, el Estado también opera bajo esta lógica de cálculo monetario, pero además realiza un cálculo en función de los costos políticos de sus actos. Al respecto, es posible observar cómo las instituciones del Estado deben responder a los cálculos políticos que realizan sus autoridades de turno en el gobierno, lidiando e interactuando con grupos que tienen intereses específicos y de la más diversa índole, entre los cuales están los grupos de grandes empresarios, los gremios profesionales, las organizaciones sociales y los partidos políticos, entre otros, cuyo objetivo es mantener la cohesión social y perpetuarla, ojalá en posteriores regímenes de gobierno y en beneficio de su coalición, partido, etc.

En este punto pareciera emerger una forma/cálculo supracontenedora que es la económica. Todos los actores, y específicamente el Estado, operan en estas formas de cálculo económico debido principalmente al modelo socioeconómico instaurado en Chile, donde la primacía del mercado y su rol regulador de la sociedad realza estos tipos de cálculos.

Sin embargo, a pesar de que el cálculo económico es fundamental para las acciones y decisiones que se manifiesten en el territorio, también están supeditadas por otros tantos cálculos. Por ejemplo, para que el Estado estime un buen gobierno, debe asegurar el empleo, en consecuencia, sus cálculos con respecto al nivel de empleabilidad de un territorio serán importantes al momento de decidir la instalación de un proyecto productivo.

Por otra parte, al interior del Estado los cálculos de servicios específicos estarán dados por la capacidad de investigación y tecnologías que se tengan; en este sentido, un ejemplo relevante es el de la Dirección General de Aguas, que ha de calcular la cantidad de metros cúbicos de agua por segundo que corresponden a cada acción de agua, y cuyos derechos de aprovechamiento ellos otorgan. Este cálculo es de trascendental importancia, pues debe estimar el equilibrio y la capacidad de carga de una cuenca. En este sentido, vale la pena mencionar que existe en la región, un caso emblemático de "error de cálculo": en la cuenca del Copiapó se otorgaron acciones de agua que superaban en un $400 \%$ la capacidad de recarga del caudal, por lo que se alteró el equilibro hídrico de la cuenca (Delegación Presidencial RRHH, 2015).

Otro aspecto que observar, son los cálculos de compensación que estipulan las empresas debido al impacto que podrá tener la ejecución de uno de sus proyectos. Cuando una empresa se instala, comienza una campaña de responsabilidad social, que la mayoría de las veces se traduce en compensaciones o "aportes" a las comunidades locales. La empresa debe calcular entonces cuánto y a quién aportará para obtener la "licencia social".

Del otro lado, y como respuesta a lo que las empresas ofrecen, las organizaciones sociales y los individuos han de valorar sus costos y beneficios. Ante la ausencia de acuerdos, normalmente exponen sus demandas en contextos como actos electorales, marchas de protesta, espacios públicos de diálogos o en negociaciones directas y privadas con la empresa, etc.

f) Tácticas. Son las acciones, definidas de antemano por cada actor, destinadas a obtener un objetivo. Atendiendo efectivamente a los ejercicios de poder que obran en un contexto especifico, es posible señalar que se ha de observar a cada actor en función de las prácticas discursivas y extra discursivas que realiza en función de sus objetivos. Del mismo modo, cada actor puede realizar una práctica discursiva o extra discursiva que no necesariamente esté en coherencia con las prácticas de la institución a la que representa, por lo cual se vuelve un poco más complejo determinar los fines u objetivos de cada acción y de cada institución. Por ejemplo, 
existe un evidente conflicto de intereses cuando una autoridad política se declara a favor de la aprobación de un proyecto que potencialmente afectará al medio ambiente y al agua, cuando recibe al mismo tiempo aportes monetarios en el marco de la responsabilidad social empresarial de dicha empresa.

Se esperaría que las prácticas políticas de cada autoridad tengan como objetivo el bien común de los ciudadanos o de la población. Entendido así, sus prácticas discursivas y extra discursivas se apoyan en objetivos concretos, mediados por cálculos económicos específicos como los del empleo, por lo tanto se comprenderá que sus decisiones están mediadas por la valoración de costos y beneficios de un proyecto (por ejemplo minero), en donde el impacto al medio ambiente y al agua es un costo, pero también proveerá de múltiples beneficios. En función de esto, las autoridades políticas acentúan sus acciones tácticas con el fin de seducir a la población con los beneficios del proyecto, tratando de ocultar y/o asumir los costos como parte ineludible del crecimiento económico y de la forma de "desarrollo minero" de la región. Otro punto interesante, es el papel de actor "neutro" que muchas veces asumen las autoridades políticas del Estado frente a lo que consideran ellos un "problema entre privados", que deben resolver entre ellos como consumidores en un mercado del agua.

Por su parte, las empresas (mineras) ostentan una serie de tácticas que se pueden observar en sus políticas de responsabilidad social empresarial. En ellas se establecen las prácticas discursivas y extra discursivas que deben realizar los empleados, orientadas a alcanzar el objetivo de que la empresa reciba la denominada "licencia social para operar" (LSO) o la aprobación para ejecutar sus tareas sin problemas. En ese contexto se presentan una serie de elementos discursivos que tratan de resaltar, ante las comunidades, los beneficios del proyecto, además de ofrecerles una serie de regalías que equivalen a compensaciones por los problemas que ya se presentan, o que pudieran surgir debido a la instalación y funcionamiento de un proyecto que demandará gran cantidad de agua.

Del otro lado, las organizaciones de la sociedad civil despliegan a su vez otras tácticas, que constituyen relevantes elementos de estos juegos de poder que se dan en el caso del agua. El Código de Aguas establece y señala la institucionalidad y las condiciones claras y vigentes de cómo actuar en esta dimensión. Dicho esto, las tácticas de las organizaciones varían en función de la complejidad del objetivo a alcanzar: cuando existe la necesidad de oponerse a un proyecto productivo que implica un uso intensivo del agua, las organizaciones despliegan acciones para contar con el apoyo de los poderes del Estado, así como de otras organizaciones, etc.; pero cuando se trata de objetivos monetarios, la táctica es simplemente acceder a negociar directamente con la empresa.

En general, todas las acciones ejecutadas por los diferentes actores, y que sean consideradas tácticas, se encuentran en lo permitido por las leyes y normas, pero dichas acciones están en constante evolución y prueba, para alcanzar los objetivos pretendidos por cada actor en cuestión.

\section{Población objetivo o pensar "como meta a la población"}

Enunciados estos seis elementos constitutivos de la gubernamentalidad, hay que entender que "este conjunto constituido que permite ejercer esta compleja forma de poder" (Foucault, 2006, p. 136), tiene como meta la población. Se ha de señalar que "la población", en estos casos, es una variable compleja de incorporar en los análisis de gubernamentalidad que aquí se plantean.

Al decir que este ejercicio tiene como meta la población, son varias las escalas con las cuales se puede estar haciendo referencia a "la población": por una parte, la población 
nacional (17 millones de habitantes), que es la más relevante en un país centralizado como Chile, y en donde la actividad minera representa el principal aporte al PIB nacional, pero cuya población se encuentra instalada principalmente en la zona central del país. Por otra parte (y aquí sería la población de escala regional), y recordando lo ya mencionado, está la población de la región de Atacama, que bordea los 290.000 habitantes (INE, 2012), lo cual representa una mínima parte del total de chilenos. Esto plantea un elemento fundamental cuando autoridades de nivel nacional, realizan acciones que favorecen la instalación de proyectos mineros de gran envergadura, aun cuando estos representan una afectación a los cauces de delicado equilibrio ecológico, como los de la región de Atacama. La población de esta demarcación se caracteriza, según el Índice de Desarrollo Regional, por tener bajos niveles de escolaridad y de salud (IDERE, 2016), a lo que se suman altos niveles de desempleo y una considerable población flotante, pues se movilizan constantemente debido a las faenas mineras. En general, se habla de una población habituada a condiciones adversas de vida, en comparación con el resto de la población nacional.

En este orden de ideas, las instituciones, procedimientos, análisis, reflexiones, cálculos y tácticas, serán distintas dependiendo de la población objetivo: nacional o regional. Cuando se analiza el ejercicio de poder a nivel nacional, se observa que hay instituciones formales claves que se derivan del poder ejecutivo central y sus respectivos ministerios (Minería, Energía, del Medio Ambiente); así como las instituciones del poder legislativo (Senado y Cámara de diputados) y la Comisión Nacional del Medio Ambiente. Dichas instituciones son relevantes dado que en ellas se discuten proyectos de ley que implican competencias medioambientales y que permiten u obstruyen la implementación de distintos proyectos productivos específicos. Por otra parte, el Comité de Ministros, instancia específica que se convoca cuando se presentan proyectos de profusa complejidad, y para los que se considera que las competencias regionales no son suficientes, es también un ejemplo de cómo, a partir de la población objetivo en cuestión, en este caso nacional, van a operar distintas instituciones, discursos, tácticas, análisis, reflexiones y procedimientos.

A escala regional, se observa que las tácticas y estrategias de las empresas están dirigidas principalmente a las poblaciones regional, local, comunal y subcomunal, ante quienes despliegan acciones en orden de alcanzar su aprobación para la ejecución de proyectos locales.

\section{Economía política}

Para Foucault, la economía política es la "ciencia que capta la red continua y múltiple de relaciones entre población, el territorio y la riqueza" (Foucault, 2006, p. 133). Deviene entonces, en el saber fundamental mediante el cual se articulan esta serie de elementos orientados al gobierno de la población.

Considerado este marco, se deriva entonces que, conocer y definir la población objetivo, tener claras las principales riquezas de las que se dispone y seleccionar el territorio, constituyen la fuente del saber mediante el cual se describirán y argumentarán las decisiones en torno a los gobiernos de los bienes naturales y el agua.

Si anteriormente se mencionó la importancia de identificar la población objetivo mediante su análisis y determinación de escalas, también es necesario considerar las riquezas y el territorio, pues la relación de todos estos elementos construye el saber principal de la gubernamentalidad. De acuerdo con el caso de estudio, encontramos:

- Las riquezas: factor fundamental para entender la gubernamentalidad. Hay que anotar que estas son catalogadas como tal, de acuerdo con el contexto histórico que se observe: en la actualidad, la mayor fuente de riquezas de 
Chile son las materias primas mineras (cobre, oro, plata), y el capitalismo global integrado posiciona al país como un proveedor de primer orden mundial. En el pasado la riqueza era el salitre y en un futuro será el litio. En resumen, las riquezas del país están representadas por estas materias primas.

En este orden de ideas, se denota claramente que, para el país, el agua no representa una riqueza tan importante como los recursos minerales, pero cada vez son más las opiniones contrarias y las polémicas al respecto. Hoy el agua es un recurso visto como insumo de la actividad minera ${ }^{1}$. Tanto es así, que su uso prioritario no es ni siquiera para el consumo humano, y esto se refleja en que, para abastecer de agua potable a la población, hoy en día se construyen plantas desalinizadoras de agua, mientras que, para las actividades mineras, se utilizan las aguas dulces de la región. Aquí cabe mencionar la polémica actual respecto a si es posible considerar el agua como una riqueza en términos económicos, como una mercancía transable en el mercado, como un recurso insumo para la minería o como un capital eje de inversión de proyectos productivos, mientras se levantan voces reclamando que se le considere (al agua) como derecho humano y como bien común natural.

- El territorio: la condición geográfica de esta región es desierto y presenta escases de recursos hídricos a pesar de que la atraviesan tres cuencas, que configuran el paisaje y el metabolismo social, pues albergan los asentamientos de la población humana. Es una región que por décadas ha sido terreno de explotación minera, por lo que ha adquirido características singulares: es parte de un territorio nacional mayor y tiene una función específica (minería); su población, que acepta y se auto identifica con esta denominación minera, también asume que un "elemento vital" para su existencia esté bajo gobiernos de mercado, aunque esta forma de gestión pueda atentar contra la salud debido a la mala calidad del servicio de la empresas sanitarias y a la escasez del agua potable.

En síntesis, entender la economía política como saber principal para el ejercicio de este complejo poder gubernamental en torno a la cuestión del agua, es fundamental para describir cómo las percepciones en torno a la población, las riquezas y el territorio, han ido cambiando con el paso del tiempo, desembocando paulatinamente en la actual situación de estrés hídrico que sufre la región, en un contexto hegemónico del modelo neoliberal.

\section{Dispositivos de seguridad}

Vinculado con lo anterior, existen tecnologías estratégicas de gobierno que se configuran en específicos dispositivos de seguridad para el gobierno del agua, los cuales representan una herramienta fundamental para ejercer el poder. Entender cómo operan las instituciones, en función del gobierno de los recursos naturales, y la gestión de la ciudadanía en estos procesos, permite observar -a nivel global- cómo las instituciones públicas y privadas generan un conjunto de estrategias, programas y dispositivos de poder altamente tecnológicos, y su expresión local se manifiesta de acuerdo con el contexto territorial específico en el que se desplieguen. El Banco Mundial, Naciones Unidas, gobiernos nacionales de turno y empresas transnacionales, trabajan coordinadamente para generar políticas, programas, planes y proyectos de "gestión del recurso hídrico" o "planes de gobernanza del agua".

En este punto es interesante la función del Estado y sus instituciones, cuando los dispositivos de poder fundamentales que dan cabida los distintos mega proyectos que utilizan una gran cantidad de agua en la región, son posibles gracias a que tienen su apoyo en un régimen de discursos que están enunciados en diferentes documentos que conforman una memoria de pautas de "conducción de conductas" respecto a lo ambiental y al agua particularmente. Estos documentos y registros son las "reglas del juego" 
y están principalmente enunciados en las leyes y códigos respectivos, tales como el de minería $\mathrm{y}$ agua, $\mathrm{y}$ es en función de ellos que se desarrollan las acciones de los actores involucrados. En esta misma línea, la Ley sobre Bases Generales del Medio Ambiente (Ley 19.300 de 1994), también constituye un sustento importante de estos enunciados, que se enmarcan en lo que se ha denominado como el "paradigma de la capitalización de la naturaleza" (Leff, 1995), que restringen las posibilidades de entender el agua y el medioambiente como bienes comunes.

Así, estas normas jurídicas son las que dan vida a los organismos del Estado, principalmente al Ministerio del Medio Ambiente y a la Dirección General de Aguas, que son a los que acuden, tanto empresas como organizaciones de resistencia, para manifestar sus acciones: las empresas tratarán de ceñirse a los mandatos del Estado en el marco del modelo neoliberal, y, por el contrario, las organizaciones le demandarán al Estado el cuidado del medioambiente y el agua.

Un análisis micropolítico de la gubernamentalidad ofrece una mirada del rol del Estado, como "distintos organismos en coordinación para ejercer diversas fuerzas" en función de los intereses de los "actores privados", que se dan cita en estas formas de gobierno. En la región de Atacama, la gubernamentalidad en torno al agua va a tomar distintas configuraciones en función de los territorios en los que se manifieste. Cada territorio va a tener sus especificidades; un ejemplo de ello es el caso de la cuenca del río Huasco, donde el rol de la Corporación Nacional de Desarrollo Indígena (CONADI) ha sido otorgar la validez y la personalidad jurídica a ciertas organizaciones indígenas (que apoyan a las empresas) y negárselas a otras (en resistencia), a través de una serie de mecanismos burocráticos que generan una forma "sujeto diaguita" diseñada por el Estado. Estas acciones es posible observarlas en todos los organismos estatales, que las adoptan dependiendo del territorio donde operan en torno a la gestión y administración del agua.
La noción de dispositivo parece más adecuada para ilustrar estos fenómenos micropolíticos y territoriales, en donde, en una compleja relación de actores, cuyas prácticas discursivas y extra discursivas son dinámicas y cambiantes, es más difíciles de distinguir dicotomías como actores públicos/actores privados. Como resultado, hay lugar a múltiples configuraciones de lo que podríamos llamar un dispositivo de seguridad del agua conformado por las instituciones que ejercen el poder en lo micropolítico y territorial.

\section{Gobierno de sí y de los otros: subjetivación, lucha y resistencia}

Foucault plantea que la gubernamentalidad se da en el "encuentro entre las técnicas de dominación ejercidas sobre los otros y las técnicas de s'́" (Foucault, 1994, p. 785).

Si entendemos el análisis anterior a la luzde los seis componentes de la gubernamentalidad, se observa que corresponden principalmente a las técnicas de dominación ejercidas sobre los otros, pero que cuando son aceptadas mediante las técnicas que ejerce sobre sí cada persona, damos cuenta de una relación de poder compleja como es la gubernamentalidad.

En esta conceptualización teórica, la analítica del poder de Foucault va estar enfocada, en última instancia, a la problemática del sujeto y la forma histórica que adopta: forma-sujeto. Foucault niega la posibilidad de una naturaleza o esencia del sujeto cartesiano, se va a concentrar en una historia del sujeto, o más específicamente en los modos de subjetivación (Castro, 2004). En esta construcción de formas del sujeto, es interesante observar cómo los propios sujetos involucrados en los ejercicios de poder en torno al gobierno del agua asumen y construyen su propia subjetividad.

Los modos de subjetivación son el resultado de los mecanismos mediante los cuales se objetiviza el sujeto y que el conocimiento 
constituye mediante "juegos de verdad" en la relación entre saber y poder. Estos modos de subjetivación, según Foucault, son básicamente tres: en primer lugar, la subjetivación de las ciencias, que construye un sujeto determinado como por ejemplo el "sujeto productivo" y posteriormente el "sujeto emprendedor", conceptos acuñados recurrentemente por la economía política. En el contexto de la región de Atacama, los discursos político-formales han instaurado la denominación de un "sujeto minero".

En segundo lugar, encontramos las prácticas divisorias como modo de subjetivación, donde el sujeto es dividido en sí mismo y de los otros; Foucault se refiere a las distinciones sano/enfermo y loco/cuerdo, que analizó en sus estudios (Morey, 2014). A efectos del presente trabajo, este modo de subjetivación concuerda con lo que el modelo de aguas en Chile establece, al relacionar y clasificar entre un consumidor/no consumidor, accionista/no accionista de agua (campesinos), accionista mayoritario/ accionista minoritario, cliente/no cliente, entre otros. Así mismo, es interesante lo que hace el proceso de participación ciudadana cuando establece quién puede participar o no en una evaluación de proyecto, y denomina organizaciones formales/no formales. Estas mismas prácticas divisorias pueden darse sobre el sujeto mismo: al hablar del tema del agua, estamos frente a un sujeto que es ciudadano/cliente; ciudadano en torno a las decisiones sobre el agua, o sea con un rol político asignado, y cliente, en tanto que consumidor del agua.

El tercer modo de subjetivación es entendido como las "formas de actividad sobre sí mismo", es decir, cómo el sujeto actúa en función de su constitución como sujeto. En el contexto del tema del agua que nos ocupa, se entiende que el modelo del agua establece claramente un rol de agente propietario de un bien de consumo (el agua), situación que subjetiviza en propietario y consumidor a los sujetos. Este modo de subjetivación ha generado gran resistencia por parte de otros grupos y sujetos en la región de Atacama y en Chile en general.
En este sentido y siguiendo a Foucault, quien plantea que la resistencia va a ser parte constitutiva de los ejercicios de poder ("donde ha poder hay resistencia"), no se entiende un poder sobre algo que no lo resista; no hay poder sin resistencia, no se necesita. Por lo tanto, las formas de resistencia van a ser permanentes (con esto se aleja de entender la revolución como única forma de contrapoder). Para el filósofo, las resistencias se manifiestan constantemente y de manera dinámica y dispersa; para realizar un análisis desde la perspectiva de la resistencia, Foucault afirma que habría que saturarse en el análisis de las luchas (Castro, 2004).

Las luchas para Foucault (1994), son de tres tipos: "contra la dominación: étnica, social, religiosa; contra la explotación: separación de individuos de lo que producen; y contra las formas de sujeción: que vinculan al sujeto consigo mismo, y aseguran su sujeción a los otros" (p. 247). Estas tres formas operan y funcionan complementariamente, pero la última es de especial interés cuando se estudian las resistencias a los modos de subjetivación que se han instalado respecto al gobierno del agua.

En este sentido, es posible entender cómo los sujetos adquieren una posición en función de la construcción de subjetividad. Por ejemplo, posturas como "el ciudadano responsable socialmente", "el ecologista", "el ambientalista", que, si bien son categorías simplificadas al extremo, permiten dilucidar las posiciones desde donde discurren los discursos de los propios sujetos. Así, la subjetivación de una persona en usuario o cliente, respecto al recurso hídrico, va a estar en la vanguardia de las prácticas discursivas de los dispositivos de poder de la gubernamentalidad del agua.

Esta idea es similar a la hipótesis de $\mathrm{Mu}-$ rillo (2015), en la que plantea que el "neoliberalismo intensificó los procesos de subjetivación centrado en la construcción del yo como objetivo central de sus tácticas" (p. 12). En este sentido, es interesante señalar que el ejercicio de 
poder en torno al agua, a través de la gubernamentalidad, se concentra en la transformación del campesino o regante ${ }^{2}$, en usuario o cliente del agua, lo que aun despierta resistencias en algunas organizaciones. Cabe señalar que también se observan menores resistencias y mayor sometimiento a estas nuevas lógicas de ejercicio de poder y producción de sujetos.

Respecto a las resistencias de menor intensidad, es interesante observar que cuando se secó la corriente superficial del río Copiapó en el año 1997, no se produjeron mayores cuestionamientos a las causas de este fenómeno. Por otra parte, y anterior a este suceso, durante el proceso de privatización y regularización de derechos de aprovechamientos en las cuencas regionales, las manifestaciones de resistencias y oposición estaban muy inhibidas debido al régimen represivo de la dictadura cívico-militar (Sanz, 2016); caso contrario ocurrió en Bolivia durante la denominada "guerra del agua".

En el presente es posible observar la creciente cantidad de movimientos socioambientales de resistencia en la región de Atacama, los cuales, ante la instalación de megaproyectos mineros (Pascua-Lama, El Morro); energéticos (Central Termoeléctrica Punta Alcalde), y agroindustriales (Agrosuper S.A.), exaltan discursos como "el agua es vida", "no más zonas de sacrificio", "no más muertes por cáncer", entre otros. Se evidencia una reivindicación de la importancia de la vida ante los peligros que representan estos proyectos, riesgos que afectan directamente la salud de los habitantes atacameños, dando paso a una resistencia a la subjetivación del ser humano como máquina productiva y parte del engranaje del sistema económico imperante, y posicionando la vida como valor predominante en la subjetivación de las personas (sujeto portador de vida), con el reajuste de lo que significa el agua para estos procesos vitales.

\section{Algunas conclusiones}

Volviendo entonces a la pregunta de cómo puede contribuir la filosofía política propuesta por Michel Foucault a la comprensión, explicación y transformación de la crisis hídrica actual en el norte de Chile, es posible señalar:

La caja de herramientas posee una potencialidad polisémica que permite analizar una gran variedad de elementos que deben ser ajustados en función del énfasis del "obrero" que utilice estas herramientas. En este ajuste y aplicación, los distintos componentes cobrarán mayor o menor importancia en función del caso que se estudie. Así, la importancia y potencia del análisis de los ejercicios de poder se debe a que aglutina a las distintas y específicas instituciones, procedimientos, análisis, reflexiones, cálculos y tácticas involucradas en esta red de poder. La noción de "caja de herramienta" permite construir conocimiento sin necesariamente adscribirse a la rigidez de un marco teórico preestablecido a los fenómenos observados, más bien plantea guías y herramientas para la construcción de un conocimiento enriquecido.

En cuanto a la construcción de subjetividad, es fundamental para entender el desarrollo del caso; la efectividad de los ejercicios de gubernamentalidad del ambiente y el agua ejercidos por la institucionalidad pública y la empresa sobre la población, son irrefutables, pues han logrado que gran cantidad de personas espere que la minería extractiva sea la forma de "desarrollo", "crecimiento" y "empleo", que les permita vivir mejor. El discurso del minero y de la región minera ha calado hondo, tanto así que se naturaliza esta forma de vida en el norte del país y pareciera ilusorio plantear otras alternativas, es por ello que las organizaciones de resistencia se manifiestan contra estas formas de gobiernos económicos y sus políticas, que naturalizan lo ambiental y el agua como un recuso.

Esto es resultado de lo que Guattari (2015) plantea en su concepto de capitalismo mundial integrado (CMI), indicando que es productivo, económico y subjetivo. Cuestión que aborda de cierta forma Murillo (2015), quien afirma que el neoliberalismo es una racionalidad de gobierno 
que considera fundamental la subjetividad y su construcción. El rol de la minería como el "sueldo de Chile" coinciden con el modelo neoliberal como forma de gubernamentalidad. $\mathrm{Su}$ principal manifestación en el territorio de Atacama es que, desde hace años, la población local ha normalizado el potencial riesgo de contaminación y escasez que representa el actual uso del agua.

Vinculado con lo anterior, en la región de Atacama la cuestión del agua ha generado resistencias, pues se denuncian potenciales agresiones a las condiciones de vida y a la vida misma de la naturaleza y de las poblaciones del valle del Huasco. Es decir, algunas asociaciones o grupos se resisten a autoindentificarse como "mineros", "emprendedores" y/o "proveedores de servicios"; estos movimientos también se resisten contra la subjetivación de la población en función de "formas sujeto" que beneficien a los intereses de la empresa.

Es interesante observar cómo las acciones de los movimientos y organizaciones de resistencia se inclinan hacia sus conciudadanos en función de mecanismos de contra subjetivación; $\mathrm{y}$ frente a los mecanismos estatales y empresariales descritos.

En cuanto a la territorialidad, existe la posibilidad de entender la gubernamentalidad como una forma de gobierno biopolítica, que tiene a la economía política como saber principal, la cual articula la integración de las riquezas, población y territorio, y permite relevar el aspecto territorial en la analítica del poder. Esto evidencia que para entender lo que significa el poder y gobierno del agua en el desierto, la institucionalidad pública ambiental ha de considerar que el país tiene diversidades geográficas, que marcan diferencias al momento de pretender gestionar el ambiente en todo el territorio nacional de manera homogénea.

La gubernamentalidad, al momento de ser aplicada a contextos espacio-temporales mi- cro en torno al agua, opera de manera flexible y potente, se sitúa y orienta hacia una "historia del presente", sin perder su alcance más global e histórico pues es parte de ella. De acuerdo con el objeto de estudio, se observa que el caso del agua en el desierto de Atacama es un campo en disputa, donde la analítica del poder permite vislumbrar multiplicidad de actores, instituciones, racionalidades, saberes, poderes/resistencias y subjetivaciones en permanente desarrollo y que operan sobre lo ambiental y el agua. Es una oportunidad de reterritorializar los análisis, salir de las explicaciones universales y analizar el poder en contextos geográficos específicos, en una especie de gubernamentalidad territorial de los bienes comunes naturales.

El concepto de gubernamentalidad aplicado a la cuestión de agua, parece atravesar las barreras disciplinares para abordar sus fenómenos; como buena filosofía, trata de abordar los temas de manera integral y profunda y plantea un cuestionamiento crítico a la forma en que se construye el conocimiento y sus herramientas. Desde este punto de vista, suscita una discusión respecto a las bases epistemológicas de la construcción del conocimiento y el carácter de verdad que se construye históricamente (regímenes de verdad). En este camino, Swyngedouw (2015), con su "ciclo hidrosocial", ha avanzado hacia lo transdisciplinar al plantear una analítica del poder entre lo social y el agua. Si bien sus análisis responden a contextos más urbanos, son de utilidad para posibles análisis en territorios rurales como la región de Atacama.

El enfoque de gubernamentalidad aplicado a cuestiones ambientales y de bienes comunes naturales, puede entenderse como una crítica a la centralidad del sujeto y su pretensión de objetividad y control sobre lo que estudia. En este sentido devela el ejercicio de poder que significa todo saber, y específicamente investiga estas manifestaciones de la cultura occidental moderna de la cual somos herederos. Sin embargo, resta establecer cómo este concepto 
teórico, acuñado en otro tiempo y lugar, permite establecer la historia de la gubernamentalidad de los bienes comunes naturales en nuestra América, concediendo la creación, desde América Latina, de una crítica decolonial con la que se censura el eurocentrismo académico y la forma de construir conocimiento (Castro-Gómez \& Grosfoguel, 2007).

Para estudiar lo ambiental (o socio-ecológico), son valiosos algunos conceptos de la filosofía política de Foucault, como la biopolítica; la gubernamentalidad; la arqueología y la genealogía; los discursos y la noción de "medio". Concretamente, el concepto de gubernamentalidad aúna otra serie de conocimientos que permiten, a partir de un enfoque micropolítico y territorial, entender las relaciones que se han venido configurando en el tiempo (1973-2005) y en el espacio (local/global) en Chile y particularmente en la región de Atacama con respecto al problema del agua.

El enfoque de gubernamentalidad permite determinar las causas de la actual situación del agua en el desierto de Atacama (particularmente) y en Chile. Adicionalmente, facilita estimar el devenir histórico de las relaciones de poder en la heterogénea red de actores reseñada en el presente artículo, y cuya construcción de subjetividad ha sido tan exitosa, que se ha naturalizado por completo, aun cuando la situación hídrica sea un tema crítico para la subsistencia de la población. Habría que abordar los mecanismos de subjetividad necesarios para revertir dicha situación.

\section{Notas}

1 Cuestión que tanto el Código de Aguas como el de Minería permiten. Actualmente se discute en el Congreso que el uso prioritario del agua sea para el consumo humano.

2 Persona que utiliza el agua para el riego, designación utilizada en la normativa jurídica.

\section{Referencias}

Agrawal, A. (2006). Environmentality. Technologies of the government and the making of subjects. Durham: Duke University Press.

Bakker, K. (2010). Privatizing water. Governance failure and the world's urban water crisis. Ithaca: Cornell University Press.

Bakker, K. (2013). Constructing 'public' water: the World Bank, urban water supply, and the biopolitics of development. Environment and Planning D: Society and Space, 31 (2), 280-300. doi: 10.1068/ d5111

Castro, E. (2004). El vocabulario de Michel Foucault. Un recorrido alfabético por sus temas, conceptos y autores. Buenos Aires: Prometeo; Universidad Nacional de Quilmes.

Castro-Gómez, S. (2010). Historia de la gubernamentalidad. Razón de Estado, liberalismo y neoliberalismo en Michel Foucault. Bogotá: Siglo del Hombre Editores; Pontificia Universidad JaverianaInstituto Pensar; Universidad Santo Tomás de Aquino.

Castro-Gómez, S., \& Grosfoguel, R. (2007). El giro decolonial: reflexiones para una diversidad epistémica más allá del capitalismo global. Bogotá: Siglo del Hombre Editores; Universidad Central, Instituto de Estudios Sociales Contemporáneos y Pontificia Universidad Javeriana, Instituto Pensar.

Centro de Estudios Públicos -[CEP]. (1992). El ladrillo: bases de la política económica del gobierno militar chileno. Santiago de Chile: Autor. 
Comisión Económica para América Latina y el Caribe -[CEPAL]. (2015). Análisis, prevención y resolución de conflictos por el agua en América Latina y el Caribe (Serie Recursos Naturales e Infraestructura $\mathrm{N}^{\circ}$ 171). Santiago de Chile: Naciones Unidas.

Cortés, M. (2010). La Muerte Gris de Chañaral. El libro negro de la división Salvador de Codelco Chile. Chañaral-Chile: Impresos Plaza.

Darier, E. (1999). Foucault and the environment. In., edi- tor, Discourses of the environment. Malden, PA: Blackwell.

Delegación Presidencial para los Recursos Hídricos. (2015). Política Nacional de Recursos Hídricos. Chile: Ministerio del Interior y Seguridad Pública. Recuperado de http://www.interior.gob. cl/media/2015/04/recursos_hidricos.pdf

Duarte C., \& García A. (2013). Derechos humanos en la Región de Atacama: Migraciones y medio ambiente. Revista Trabajo Social, 84, 19-30.

Foucault, M. (1994). Dits et écrits IV. Paris: Edición Gallimard.

Foucault, M. (2006). Seguridad, territorio y población. Curso en el colege de France: 1977-1978. Buenos Aires: FCE.

Foucault, M. (2007). Historia de la sexualidad II El uso de los placeres. Buenos Aires: Biblioteca Nueva.

Foucault, M. (2015). Historia política de la verdad. Una genealogía de la verdad: breviarios de los cursos del College de France (Ed. y Trad. J. Alvarez-Yaguez). Madrid: Biblioteca Nueva.

Gajardo, A. (2014). Etnicidad, reemergencia indígena y conflicto minero. El proyecto
Pascua Lama, [el Estado] y el proceso de reetnificación de los Diaguitas del Huasco Alto, Chile. Anuari del conflicte social, 4, 73-105.

Guattari, F. (2015). ¿Qué es la Ecosofia? (Textos agenciados por Stéphane Nadaude). Buenos Aires: Editorial Cactus.

Harvey, D. (2005). El nuevo imperialismo: acumulación por desposesión. En L. Pantich \& C. Leys (Eds.), El Nuevo desafio Imperial (pp. 99-129). Buenos Aires: Merlin Press - Clacso.

Harvey, D. (2007). Breve historia del neoliberalismo. Madrid: AKAL.

Instituto Nacional de Estadísticas Chile (INE) CENSO 2012

Lander, E. (Comp.). (2000). Ciencias sociales: saberes coloniales y eurocéntricos. En, $L a$ colonialidad del saber: eurocentrismo y ciencias sociales. Perspectivas Latinoamericanas (pp. 4-23). Buenos Aires: Consejo Latinoamericano de Ciencias Sociales -[CLACSO].

Leff, E. (1995). Globalización, racionalidad ambiental y desarrollo sustentable. Recuperado de: http://oraloteca.unimagdalena.edu.co/wp-content/uploads/2013/03/ Globalizaci\%C3\%B3n-Racionalidad-ambiental-y-desarrollo-sustentable.-EnriqueLeff.pdf

Legg, S. (2005). Foucault's population geographies: classi- fications, biopolitics and governmental spaces. Population, Space and Place, 11, 137-56. doi: $10.1002 / \mathrm{psp} .357$

Luke, T. (1995). On Environmentality: Geo-Power and Eco-Knowledge in the Discourses of Contemporary Environmentalism. Cultural Critique, 31, 57-81. 
Machado, H. (2014). Potosí, el origen. Genealogía de la minería contemporánea. Buenos Aires: Editorial Mardulce.

Martínez, J. (2004). El ecologismo de los pobres. Conflictos ambientales y lenguajes de valoración. Barcelona: Icaria Antrazyt.

Morey, M. (2014). Lectura de Foucault / Escritos sobre Foucault. México; Madrid: Sexto Piso.

Murillo, S. (Coord.). (2015). Biopolítica y procesos de subjetivación en la cultura neoliberal. En, Neoliberalismo y gobiernos de la vida. Diagrama global y sus configuraciones en la Argentina y América Latina. Buenos Aires: Biblos.

Palomino, M. (2015). Ejercitando el poder en tiempos neoliberales: Resistencias, comodificación de la naturaleza y emprendimiento en Alto Bio Bio. En B. Bustos, M. Prieto \& J. Barton (Eds.), Ecología Política en Chile: naturaleza, propiedad, conocimiento y poder (pp. 213-235). Santiago de Chile: Universitaria.

Prieto, M. (2015). La ecología (a) política del modelo de agua chileno. En B. Bustos, M. Prieto \& J. Barton, Ecología Política en Chile: naturaleza, propiedad, conocimiento y poder (pp. 143-164). Santiago de Chile: Universitaria.

Quijano, A. (2002). Colonialidad del poder, globalización y democracia. Revista Trayectorias, 4(7- 8), 58-90.

República de Chile. Constitución Política (1980). Consejo de Estado.

República de Chile. Decreto con Fuerza de Ley 1122 de 1981. De las aguas y del derecho de aprovechamiento. Ministerio de Justicia, agosto de 1981.
República de Chile. Ley 18248 de 1983. Código de Minería. Ministerio de Minería, octubre de 1983.

República de Chile. Ley 19300 de 1994. Ley de Bases del Medio Ambiente, marzo de 1994.

República de Chile. Ley 20417 de 2010. Crea el Ministerio, el Servicio de Evaluación Ambiental y la Superintendenciadel medio Ambiente, enero de 2010.

Rutherford, S. (2007) Green governmentality: insights and opportunities in the study of nature's rule. Progress in Human Geography, 31(3), 291-307.

Sanz, C. (2006). Una fallida privatización del agua en Bolivia: el Estado, la corrupción y el efecto neoliberal. Revista Colombiana de Antropología, 42, 317-346.

Seoane, J. (2015). La reformulación neoliberal de la cuestión ambiental; una genealogía del paradigma de capitalización de la naturaleza. En S. Murillo (Coord.), Neoliberalismo y gobiernos de la vida. Diagrama global y sus configuraciones en la Argentina y América Latina (pp. 137-157). Buenos Aires: Biblos.

Squeo, F., Arancio, G., \& Gutiérrez, J. (Eds.) (2008). Características Climáticas de la región de Atacama. En Libro rojo de la Flora Nativa y Sitios prioritarios para su conservación. Región de Atacama. Chile: Ediciones Universidad de La Serena.

Svampa, M. (2013). «Consenso de los Commodities» y lenguajes de valoración en América Latina. Nueva Sociedad, 244. Recuperado de http://nuso.org/articulo/ consenso-de-los-commodities-y-lenguajesde-valoracion-en-america-latina/. 
Swyngedouw, E. (2015). Liquid power: Contested hidro-modernities in Twentieth Century Spain. Massachusetts: MIT.

Torres, R., \& García A. (2009). Conflictos por el agua en chile: el gran capital contra las comunidades locales. Análisis comparativo de las cuencas de los ríos Huasco y Rio Baker. En foco: medio ambiente, sociedad $y$ desarrollo sustentable / espacio abierto, 18(4), 695-708.

Ulloa, A. (2011). A ecogovernamentalidade e suas contradições. Ecopolítica, 1, 26-42. Recuperado de https://revistas.pucsp.br/ index.php/ecopolitica/article/view/7655

United Nations World Water Assessment Programme -[WWAP]. (2006). Water a shared responsibility (The United Nations
World Water Development Report No. 2). Paris: UNESCO.

United Nations World Water Assessment Programme -[WWAP]. (2015). Water for a Sustainable World (The United Nations World Water Development Report 2015). Paris: UNESCO.

Urkidi, L. (2008). Movimientos anti-mineros: el caso de Pascua-Lama en Chile. Revista Iberoamericana de Economía Ecológica, 8, 63-77.

Vial, C. (2016). Índice De Desarrollo Regional -IDERE 2016. Chile: Universidad Autónoma de Chile; Instituto Chileno de Estudios Municipales -[ICHEM]. Recuperado de http://www.idere.cl/wp-content/ uploads/2016/10/IDERE_2016_digital.pdf 